\title{
EFEITOS DA INCORPORAÇÃO DE PENEIRAS MOLECULARES 3A, 4A, 5A E 13X EM MEMBRANAS COMPÓSITAS DE QUITOSANA/POLI(VINIL ÁLCOOL)
}

\author{
Denice Schulz Vicentini \\ Departamento de Engenharia Mecânica - PGMAT, Universidade Federal de Santa Catarina, 88040-900 Florianópolis - SC, Brasil \\ João Cardoso de Lima \\ Departamento de Física, Universidade Federal de Santa Catarina, 88040-900 Florianópolis - SC, Brasil \\ Mauro Cesar Marghetti Laranjeira* \\ Departamento de Química, Universidade Federal de Santa Catarina, 88040-900 Florianópolis - SC, Brasil
}

Recebido em 1/12/08; aceito em 24/7/09; publicado na web em 11/1/10

\begin{abstract}
EFFECT OF MOLECULAR SIEVES 3A, 4A, 5A AND 13X INCORPORATION ON THE CHITOSAN/POLY(VINYL ALCOHOL) COMPOSITES MEMBRANES. The composite membranes prepared via incorporation of $12.5 \%$ of molecular sieves $3 \mathrm{~A}, 4 \mathrm{~A}, 5 \mathrm{~A}$ and $13 \mathrm{X}$ into chitosan/poly(vinyl alcohol) (1:1). The composite membranes were immersed in the cross-linker sulfuric acid in order to acquire high proton conductivity for applications in electrolytes in PEMCF and DMF. The influence of the molecular sieves on the structural, optical, thermal, mechanical, morphologic and protonic conductivity properties and water/methanol swelling degree of membranes were investigated.
\end{abstract}

Keywords: chitosan; molecular sieves; protonic conductivity.

\section{INTRODUÇÃO}

A utilização de células a combustível para geração de energia pode ser o alicerce de uma possível revolução não apenas energética, mas também sócio-ambiental. Dentre os vários tipos de célula a combustível reportados, aquelas que visam aplicações veiculares e/ou portáteis, na forma de membranas trocadoras de prótons (PEMFC) e de injeção direta de metanol (DMFC) utilizam membranas poliméricas (PEMs) condutoras de prótons..$^{1-6}$

A viabilidade econômica das células a combustível para as aplicações veiculares ou portáteis depende do desenvolvimento de novos materiais com alto desempenho e baixos custos, como catalisadores e PEMs. Para um bom desempenho as PEMs necessitam de propriedades específicas, como elevada condutividade protônica, baixa permeabilidade aos gases reagentes, elevada estabilidade térmica e mecânica, baixa condutividade eletrônica, estabilidade química e dimensional e níveis mínimos de umidade?

Nos últimos anos, foram desenvolvidas membranas condutoras de prótons a partir de complexos polieletrólitos (PEC) para PEMFC ou DMFC, como as membranas sintéticas de poli (éter éter cetona) sulfonada $\left(\right.$ sPEEK) ${ }^{8}$ e ácido perfluorossulfônico $\left(\text { Nafion }{ }^{\circledR}\right)^{5,9}$ e as membranas naturais de quitosana (QTS) modificada com sílica funcionalizada, ${ }^{1}$ zeólitas, ${ }^{2}$ ePVA, ${ }^{10}$ entre outras. Apesar de bem sucedida, a membrana de $\mathrm{Nafion}^{\circledR}$ apresenta algumas limitações, como o envenenamento do catalisador com $\mathrm{CO}$, requer a presença de plastificantes, geralmente água, para ativar seus níveis de condutividade, perda brusca de condutividade acima de $100{ }^{\circ} \mathrm{C}$, permeabilidade considerável ao metanol, no caso da membrana de DMFC e elevado custo. ${ }^{6,7,11}$ Desta forma, fica evidente a necessidade de desenvolver materiais alternativos para utilização em células a combustível. A QTS apresenta grande potencial devido a algumas características, tais como polímero natural e abundante na natureza, baixo custo, biodegradável, biocompatível, não-tóxico, hidrofílico e solúvel em ácidos orgânicos diluídos. ${ }^{12}$

Recentemente, Wang e colaboradores ${ }^{2}$ reportaram resultados sobre a preparação e caracterização de membranas híbridas de QTS com

*e-mail: mauro@qmc.ufsc.br zeólitas 3A, 4A, 5A, 13X, mordenita e HZSM-5 para aplicação em DMFC. Estes pesquisadores mostraram que o transporte de metanol nas membranas ocorre pela difusão, tornando o processo mais eficiente quando a zeólita de caráter hidrofóbico (maior razão de $\mathrm{Si} / \mathrm{Al}$ ) é incorporada. Também foi mostrado que a condutividade protônica diminui com o aumento da razão $\mathrm{Si} / \mathrm{Al}$, causando a diminuição do conteúdo de água nas membranas. A literatura reporta outros estudos sobre membranas de QTS trocadoras de prótons. ${ }^{13,14}$

Para um bom funcionamento da célula a combustível utilizando membranas de QTS, faz-se necessário modificar a composição e/ou adicionar outros materiais, por exemplo, plastificantes e/ou reticulantes, preparar membranas de polímero/polímero ou polímero/material inorgânico. Estas últimas são interessantes devido à contribuição para o aumento da condutividade protônica, manutenção de água em altas temperaturas e, maior sustentação mecânica. ${ }^{5}$ Neste contexto, a incorporação de aditivos inorgânicos, como peneiras moleculares (PMs), vem sendo amplamente usada em membranas poliméricas PEMs., ${ }^{2,3}$

A estrutura cristalina das PMs é constituída pela combinação tridimensional de tetraedros do tipo $\mathrm{TO}_{4}^{-}$, onde $\mathrm{T}$ representa os átomos de silício ou alumínio, unidos entre si através de átomos de oxigênio comuns. $\mathrm{O} \mathrm{Al}^{3+}$ provoca um desbalanceamento de carga, induzindo cargas negativas na estrutura que são neutralizadas por cátions de compensação, os quais juntamente com as moléculas de água, outros adsorbatos e sais, estão localizados nos poros e/ou cavidades da estrutura. ${ }^{15}$ Os sítios básicos na estrutura estão associados aos íons oxigênio. $\mathrm{O}$ número destes sítios é igual ao número de átomos de $\mathrm{Al}$ na estrutura. A razão Si/Al na composição das PMs determina o grau de hidrofilicidade das PMs. Este parâmetro é fortemente influenciado pela presença do Al. Assim, menores razão Si/Al conduzem a PMs com caráter básico e hidrofílico. ${ }^{15}$

Neste artigo, reportamos resultados dos efeitos da incorporação de PMs 3A, 4A, 5A e 13X em membranas de QTS/PVA (poli(vinil álcool)). Os efeitos foram investigados usando as seguintes técnicas: difração de raios x (DRX), espectroscopia de infravermelho (FTIR), análise térmica (TG), microscopia eletrônica de varredura (MEV), ensaios mecânicos, condutividade protônica, capacidade de troca iônica e grau de intumescimento em água e em solução de metanol. 


\section{PARTE EXPERIMENTAL}

\section{Reagentes}

Os reagentes usados na preparação das membranas foram: quitosana, com grau de desacetilação de $90 \%$ e massa molar média de $1,2 \times 10^{5} \mathrm{~g} \mathrm{~mol}^{-1}$ (determinada por viscosimetria), adquirida da Purifarma Comp. (São Paulo, Brasil); poli(vinil álcool), com grau de hidrólise entre $87-89 \%$ e massa molar média de entre $8,5 \times 10^{4}$ e $1,2 \times 10^{5} \mathrm{~g} \mathrm{~mol}^{-1}$, adquirido da Vetec (Rio de Janeiro, Brasil); peneiras moleculares 3A, 4A, 5A ou 13X (Tabela 1) adquiridas da Sigma-Aldrich (St. Louis, USA); glicerol, ácido acético glacial, ácido sulfúrico e metanol adquiridos da Nuclear (São Paulo, Brasil); água destilada; fio de platina adquirido da Degussa Brasil Ltda (São Paulo, Brasil) e, cola de prata adquirida da Joint Metal Com. Ltda (São Paulo, Brasil).

Tabela 1. Principais características fisico-químicas das $\mathrm{PMs}^{2,15}$

\begin{tabular}{lcccc}
\hline PMs & Razão Si/Al (̊) & $\begin{array}{c}\text { Diâmetro do } \\
\text { poro }(\mathrm{nm})\end{array}$ & $\begin{array}{c}\text { Moléculas } \\
\text { absorvidas }\end{array}$ & $\begin{array}{c}\text { Cátion } \\
\text { presente }\end{array}$ \\
\hline 3A & 1,0 & 0,30 & água & $\mathrm{K}^{+} / \mathrm{Na}^{+}$ \\
$4 \mathrm{~A}$ & 1,0 & 0,41 & água/metanol & $\mathrm{Na}^{+}$ \\
$5 \mathrm{~A}$ & 1,0 & 0,50 & água/metanol & $\mathrm{Na}^{+} / \mathrm{Ca}^{+}$ \\
$13 \mathrm{X}$ & 1,3 & 0,74 & água/metanol & $\mathrm{Na}^{+}$ \\
\hline
\end{tabular}

\section{Preparação das membranas}

Para a preparação das membranas foram dissolvidas 3,0 $\mathrm{g}$ de QTS em uma solução de ácido acético $2 \%$, sob agitação constante. Após completa dissolução do polímero, foram adicionados $1,0 \mathrm{~g}$ de glicerol, (plastificante) e 1,0 g de PM 3A, 4A, 5A ou 13X. A solução foi agitada continuamente por $3 \mathrm{~h}$. Paralelamente, $3,0 \mathrm{~g}$ de PVA foram dissolvidos em água com agitação e aquecimento. Os polímeros foram misturados em um único frasco e agitados durante $4 \mathrm{~h}$, para assegurar a homogeneização da mistura. O material resultante foi vertido em placas de Petri para evaporar o solvente à temperatura ambiente e posteriormente seco a $40{ }^{\circ} \mathrm{C}$ em estufa a vácuo, por $48 \mathrm{~h}$. A composição resultante das membranas de QTS/PVA apresentou uma razão de 1:1 em massa dos polímeros ( $37,5 \%)$ e $12,5 \%$ em massa de cada tipo PM. Posteriormente, as membranas foram imersas numa solução de ácido sulfúrico $2 \mathrm{~mol} \mathrm{~L}^{-1}$, por um período de $24 \mathrm{~h}$ para a reticulação iônica. A reticulação iônica ocorre entre os grupos amino $\left(\mathrm{NH}_{3}^{+}\right) \mathrm{da}$ QTS e os íons $\mathrm{SO}_{4}^{2-}$ do ácido sulfúrico. ${ }^{2,4}$ Por fim, as membranas são lavadas repetidamente com água e secas novamente. As membranas resultantes apresentaram espessuras entre 100 e $150 \mu \mathrm{m}$ e tamanho de partículas das PMs entre 4,0 e 2,0 $\mu \mathrm{m}$.

\section{Técnicas empregadas para a caracterização das membranas}

\section{Difração de raios $x$}

Os difratogramas de raio $\mathrm{x}$ foram medidos na faixa angular $2 \theta$ $=5^{\circ}-80^{\circ}$, usando um difratômetro marca Philips, modelo X'Pert, equipado com um tubo de cobre $(\mathrm{CuK} \alpha, \lambda=1,54056 \AA)$. As distâncias interatômicas $(r)$ para os primeiros vizinhos na estrutura foram estimadas usando a relação de Ehrenfest, Equação 1:

$$
r=\frac{\lambda}{E \sin \theta}
$$

onde $\lambda$ é o comprimento da radiação, $\theta$ é semi-ângulo de difração e $E$ uma constante dependente da estrutura, sendo assumida igual a 1,671.

\section{Espectroscopia no infravermelho}

A identificação dos grupos funcionais e seus possíveis deslocamentos foram avaliados usando um espectrômetro FTIR ABBBomem, modelo FTLA 2000. A faixa de comprimentos de onda usada foi de 700 a $4000 \mathrm{~cm}^{-1}$, com uma resolução de $4 \mathrm{~cm}^{-1}$. As análises de FTIR foram realizadas nas membranas com e sem tratamento prévio de secagem. O tratamento de secagem consistiu em deixar as membranas na estufa à vácuo a $50^{\circ} \mathrm{C}$ por $48 \mathrm{~h}$.

\section{Análise termogravimétrica}

A estabilidade térmica das membranas previamente secas por $24 \mathrm{~h}$ na estufa a vácuo foram medidas usando um sistema TG da Shimadzu, modelo TGA 50, e os termogramas foram registrados na faixa de temperatura de 20 a $600{ }^{\circ} \mathrm{C}$, com uma taxa de aquecimento constante de $10{ }^{\circ} \mathrm{C} \mathrm{min}{ }^{-1}$, sob um fluxo de $50 \mathrm{~mL} \mathrm{~min}^{-1}$ de $\mathrm{N}_{2}$.

\section{Microscopia eletrônica de varredura}

As micrografias da secção transversal das membranas foram obtidas usando um microscópio eletrônico de varredura Philips, modelo XL 30. Para a realização das medidas, as amostras foram fraturadas com nitrogênio líquido e recobertas com uma fina camada de ouro.

\section{Propriedades mecânicas}

Nos ensaios de tração-deformação foi determinado o módulo de elasticidade, tensão máxima na ruptura e deformação específica. Os dados foram medidos em uma máquina de ensaios universal EMICDL 2000, seguindo a norma ASTM-D882-95. As membranas foram previamente mergulhadas em água destilada por $10 \mathrm{~min}$ antes do início do ensaio, visando obter um pequeno intumescimento. Foram analisadas cinco amostras de cada membrana, a temperatura de $20 \pm$ $1^{\circ} \mathrm{C}$ e velocidade do ensaio foi de $5 \mathrm{~mm} \mathrm{~min}^{-1}$. A espessura média das PM foi obtida a partir de 4 medidas em pontos aleatórios da mesma membrana com um micrômetro Digimess, modelo Electronic Outside Micrometer, com precisão de 0,001 mm.

\section{Medidas de condutividade protônica}

As medidas de condutividade protônica foram realizadas usando um equipamento LCR Meter MXB-820, Minipa, na faixa de frequência de 100 a $10 \mathrm{KHz}$. Para realizar as medidas, eletrodos de platina foram colocados em lados opostos das membranas. Os eletrodos foram fixados usando um fio de platina e colados com cola de prata. As medidas foram realizadas com $0,1 \mathrm{~V}$ de tensão e durante as medidas a temperatura do laboratório era de $20 \pm 1{ }^{\circ} \mathrm{C}$. A condutividade protônica $\sigma\left(\mathrm{S} \mathrm{cm}^{-1}\right)$ das amostras foi calculada usando a Equação 2:

$$
\sigma=\frac{l}{A R}
$$

onde 1, A, e R são a distância entre os eletrodos (cm), a área da membrana $\left(\mathrm{cm}^{2}\right)$ e a resistência $(\Omega)$, respectivamente.

\section{Capacidade de troca iônica}

A IEC das membranas foi determinada pelo método de titulação convencional. As membranas foram imersas em uma solução 2 mol L-1 de $\mathrm{NaCl}$ por $24 \mathrm{~h}$ com intuito de substituir o $\mathrm{H}^{+}$pelo $\mathrm{Na}^{+}$. A solução foi titulada com uma solução de $0,01 \mathrm{~mol} \mathrm{~L}^{-1}$ de $\mathrm{NaOH}$, usando fenoftaleina como indicador. $\mathrm{O}$ valor da IEC foi calculado usando a Equação 3:

$\operatorname{IEC}\left(\mathrm{mmol} \mathrm{g}^{-1}\right)=\frac{0,01 \times 1000 \times V_{\mathrm{NaOH}}}{m_{d}}$ 
onde $\mathrm{V}_{\mathrm{NaOH}}(\mathrm{L})$ é o volume de solução do $\mathrm{NaOH}$ consumido na titulação e $m_{d}(g)$ é a massa da membrana seca.

\section{Absorção de água/metanol}

A porcentagem de água absorvida $(A A \%)$ nas membranas foi determinada pela razão entre a diferença de massa da membrana seca $\left(M_{s}\right)$ e após imersão em água destilada $\left(M_{i}\right)$ por $24 \mathrm{~h}$ a temperatura de $20{ }^{\circ} \mathrm{C}$. O mesmo procedimento foi repetido para a solução aquosa de metanol $12 \mathrm{~mol} \mathrm{~L}^{-1}$. Após remover as membranas da solução, estas foram secas com papel toalha para remover o excesso de solução da superfície. O valor final de absorção de água e de solução de metanol foi obtido considerando a média de três medidas determinadas pela Equação 4:

$$
A A(\%)=\frac{M_{i}-M_{s} \times 100}{M_{s}}
$$

\section{RESULTADOS E DISCUSSÃO}

\section{Difração de raios x}

A Figura 1 mostra os difratogramas de raio $\mathrm{x}$ das membranas de QTS/PVA e QTS/PVA com PMs. A membrana QTS/PVA exibe picos característicos da estrutura semicristalina da QTS em $2 \theta=11,6$; 18,$7 ; 21,3$ e $23,5^{\circ} .^{1,2,10,14}$ A literatura reporta que o pico de difração da estrutura amorfa do PVA está localizado em torno de $20^{\circ} .{ }^{10,16} \mathrm{~A}$ Figura 1 mostra que as membranas de QTS/PVA com PMs, além de apresentarem os picos da QTS/PVA, também mostram picos entre 30 e $40^{\circ}$ atribuídos às PMs puras. As membranas de QTS/PVA-3A e QTS/PVA-13X apresentaram uma diminuição na intensidade do pico característico da QTS/PVA em $18,7^{\circ}$, sugerindo que a presença das partículas de PMs interferiu no empacotamento das cadeias de QTS, reduzindo os domínios semicristalinos por efeitos eletrostáticos e por interações de ligação de hidrogênio entre a QTS/PVA e a PMs. ${ }^{2}$ As membranas de QTS/PVA-4A e QTS/PVA-5A mantiveram os picos da QTS/PVA, sugerindo que ocorreu pouca interação entre os grupos funcionais dos polímeros com estas PMs.

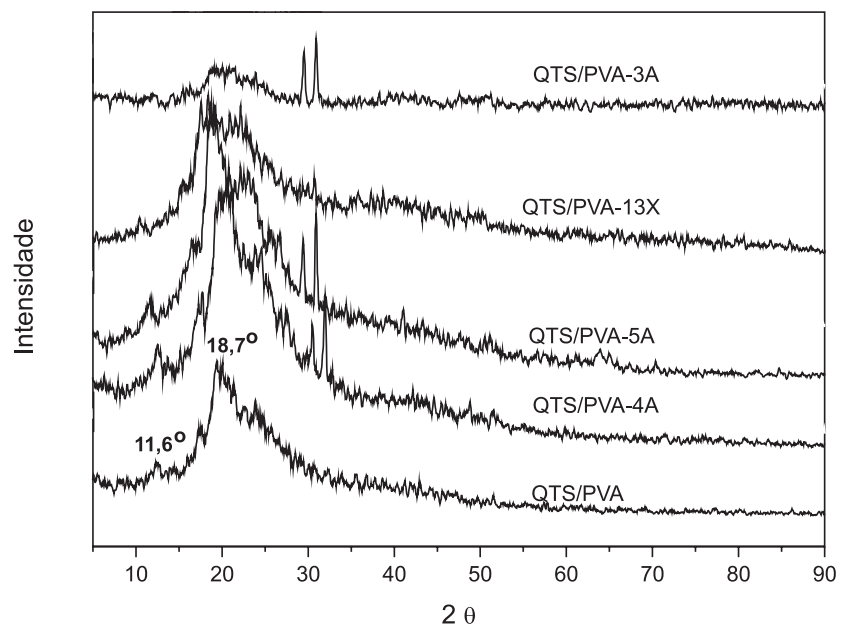

Figura 1. Difratograma de raios x das membranas de QTS/PVA e QTS/PVA com PMs

Os difratogramas de raio $\mathrm{x}$ das membranas de QTS/PVA com partículas de PMs mostram que o halo principal observado $2 \theta=$ $18,7^{\circ}$ foi modificado pela incorporação das PMs. As distâncias interatômicas foram estimadas através da Equação $1^{17}$ e os valores calculados estão apresentados na Tabela 2. A comparação entre as distâncias interatômicas evidencia que elas diminuíram após a incorporação das PMs 4A, 5A e 13X. Nesta última, a diminuição foi maior o que sugere uma maior interação entre os polímeros e a PM 13X. Para a membrana com a PM 3A, a distância interatômica calculada permaneceu a mesma da membrana de QTS/PVA. Este resultado sugere uma mistura física entre esta e os polímeros. Esta hipótese é suportada pela baixa intensidade e pouca definição dos picos presentes no difratograma de raio $\mathrm{x}$.

Tabela 2. Distâncias interatômicas médias das membranas de QTS/PVA e QTS/PVA com PMs

\begin{tabular}{lc}
\hline Membranas & $r(\AA)$ (halo principal $)$ \\
\hline QTS/PVA & 5,12 \\
QTS/PVA-3A & 5,12 \\
QTS/PVA-4A & 5,06 \\
QTS/PVA-5A & 5,06 \\
QTS/PVA-13X & 4,04 \\
\hline
\end{tabular}

\section{Espectroscopia no infravermelho}

Os espectros no infravermelho das membranas QTS/PVA e QTS/ PVA com as PMs são mostrados na Figura 2: (a) sem tratamento de secagem e (b) com tratamento de secagem. A presença de água nas membranas é indicada pelo alargamento e aumento da intensidade das bandas. As bandas características das membranas de QTS/PVA são observadas na faixa de $3600-3200 \mathrm{~cm}^{-1}$, as quais correspondem à deformação axial dos grupos hidroxila e amino. As bandas em $2928-2853 \mathrm{~cm}^{-1}$ são atribuídas ao estiramento C-H; a banda 1732 $\mathrm{cm}^{-1}$ corresponde às vibrações de estiramento $\mathrm{C}=\mathrm{O}$ do sal de acetato residual da QTS e do PVA. As absorções em aproximadente 1650 e $1568 \mathrm{~cm}^{-1}$ correspondem às deformações das bandas da amida I e amida II. ${ }^{1,2,14,18}$ As bandas localizadas próximas de 1425, 1375 e $1250 \mathrm{~cm}^{-1}$ são atribuídas aos estiramentos $\mathrm{CN}$ e, com exceção da banda de $1250 \mathrm{~cm}^{-1}$, estas bandas também podem ser atribuídas aos estiramentos $\mathrm{CH}$. As bandas em 1099 e $1042 \mathrm{~cm}^{-1}$ correspondem aos estiramentos $\mathrm{C}-\mathrm{O}$ e $-\mathrm{C}-\mathrm{OH} .{ }^{10}$

As membranas de QTS/PVA com PMs podem exibir bandas em torno de $1140 \mathrm{~cm}^{-1}$, associadas às vibrações simétricas e assimétricas do estiramento do Si-O-Al. Além destas, também podem ser observadas as bandas características dos grupos sulfônicos em 1420-1310 $\mathrm{cm}^{-1}$ e 1235-1145 $\mathrm{cm}^{-1}$, respectivamente, atribuídas às vibrações dos estiramentos assimétricos e simétricos do $\mathrm{O}-\mathrm{S}-\mathrm{O} .{ }^{1,4} \mathrm{O}$ estiramento em aproximadamente $1145 \mathrm{~cm}^{-1}$ pode estar sobreposto pela banda do Si-O-Al em $1140 \mathrm{~cm}^{-1}$ e, principalmente, pelo C-O da QTS/PVA e do plastificante (Gli).

Os espectros no infravermelho mostrados na Figura 2 foram interpretados com base nas identificações das bandas acima. Os pequenos deslocamentos das bandas localizadas em aproximadamente 1650 e $1568 \mathrm{~cm}^{-1}$ para menores frequências sugerem a existência de ligações de hidrogênio entre os grupos hidroxila e amina da QTS e os grupos hidroxilas das PMs. ${ }^{2}$ Os espectros da membrana QTS/ PVA-13X sugerem que a diminuição de intensidade observada pode ser atribuída ao aumento da razão Si/Al desta PM. Essa hipótese é corroborada pelos resultados reportados por Wang e colaboradores, ${ }^{2}$ que observaram uma redução na intensidade das bandas no espectro infravermelho de membranas de QTS com PMs proporcional ao aumento da razão Si/Al das PMs. Para membrana de QTS/PVA-3A, a diminuição de intensidade das bandas pode estar relacionada com uma possível mistura física entre a PM 3A e os polímeros, conforme sugere o difratograma de raio $\mathrm{x}$. 
a)

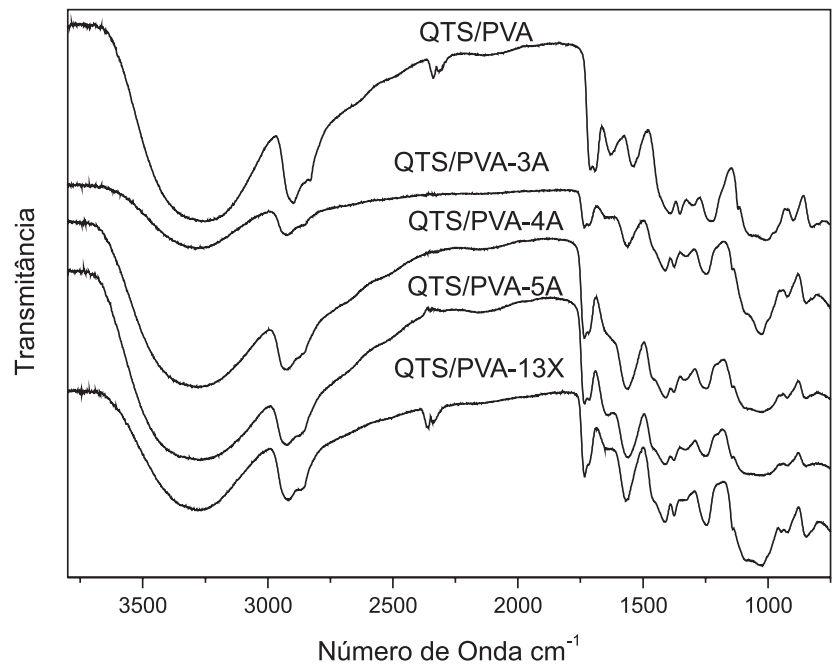

b)

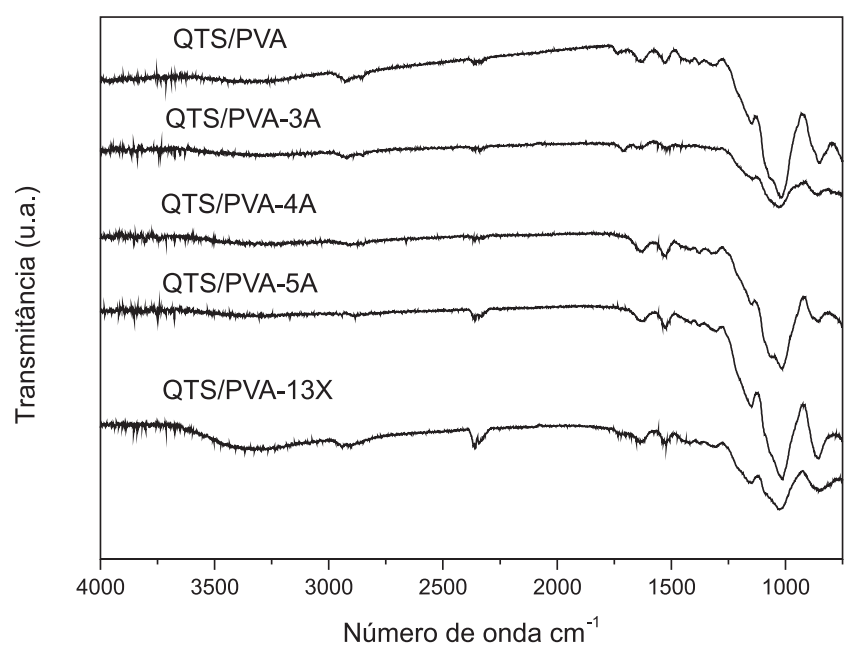

Figura 2. Espectro no infravermelho das membranas de QTS/PVA e QTS/PVA com PMs: (a) sem tratamento de secagem e (b) com tratamento de secagem

\section{Análise termogravimétrica}

A Figura 3 mostra os termogramas da membrana de QTS/PVA e as membranas de QTS/PVA com as PMs. Nesta figura observa-se que a degradação ocorreu em dois estágios. O primeiro estágio, entre 50 e $150^{\circ} \mathrm{C}$, é atribuído à perda massa de água adsorvida na superfície e a água ligada intermolecular e intramolecular nas membranas de QTS/ PVA e/ou nas PMs. Até $100^{\circ} \mathrm{C}$ predomina a adsorção superficial e, acima desta temperatura, ocorre a eliminação de água intermolecular e intramolecular. $\mathrm{O}$ segundo estágio, acima de $210^{\circ} \mathrm{C}$ corresponde à degradação da cadeia principal da QTS. Neste estágio, observa-se que a incorporação das PMs nas membranas de QTS/PVA aumenta a estabilidade térmica. ${ }^{1,2,10,14} \mathrm{~A}$ maior estabilidade térmica foi observada para a membrana de QTS/PVA-4A.

\section{Morfologia dos filmes}

A Figura 4 revela micrografias obtidas via MEV da secção transversal das membranas de QTS/PVA e QTS/PVA com PMs. Nestas micrografias pode-se observar que as membranas apresentaram aspecto homogêneo, compacto, liso, sem poros e com raros domínios de PMs. ${ }^{1,2,419}$ Pode-se concluir que a morfologia das membranas não foi afetada significativamente pela incorporação das PMs. A presença

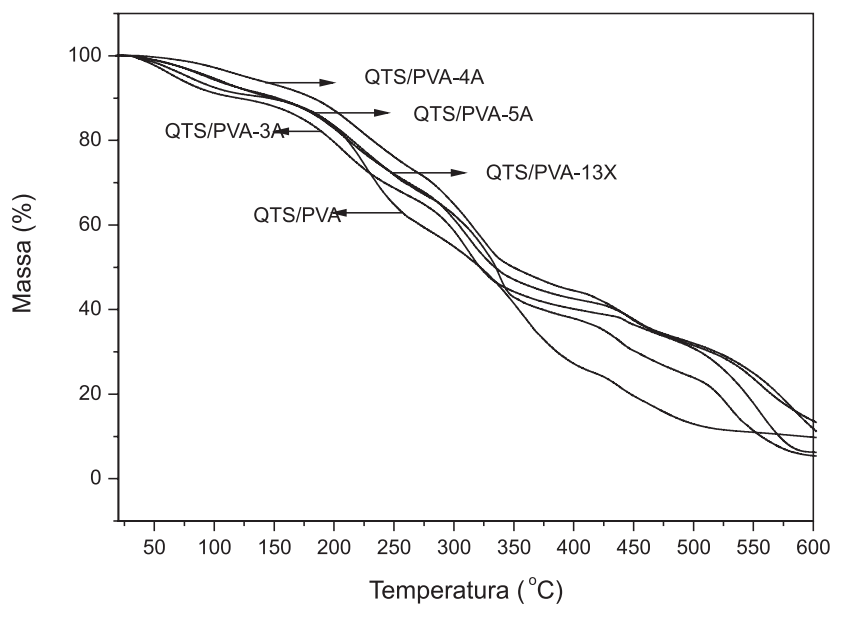

Figura 3. Análise termogravimétrica das membranas de QTS/PVA e QTS/ PVA com PMs
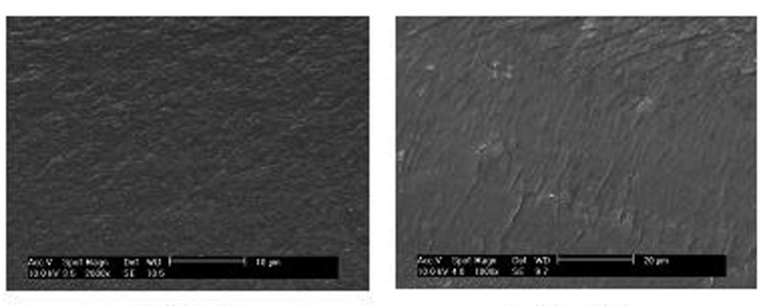

QTSPVA
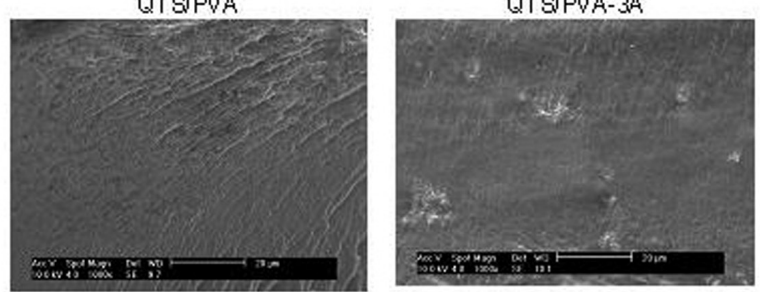

QTSIPVA-4A

QTSIFVA-5A

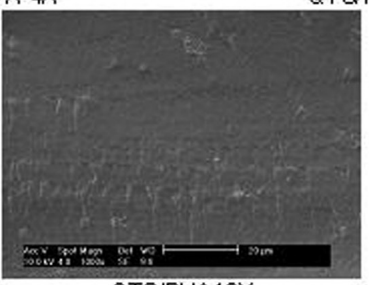

QTSIPVA13X

Figura 4. Micrografias das membranas de QTS/PVA e QTS/PVA com PMs

de raros domínios de PMs nas membranas (pequenas quantidades de fases cristalinas remanescentes das PMs) pode ser verificado através de alguns picos estreitos, vistos nos difratogramas de raio $\mathrm{x}$ das membranas de QTS/PVA com as PMs.

\section{Propriedades mecânicas}

As propriedades mecânicas foram influenciadas pela adição das PMs e principalmente, pela reticulação com o $\mathrm{H}_{2} \mathrm{SO}_{4}$ (Tabela 3). Verificou-se que as membranas apresentaram tensões máximas próximas e adquiriram um caráter rígido, diminuindo a deformação. Os resultados sugerem que os aumentos na rigidez estão associados às ligações iônicas resultantes da reticulação e das interações eletrostáticas entre os polímeros e as PMs, restringindo a mobilidade das cadeias poliméricas. Similar comportamento foi observado por Cui e colaboradores ${ }^{10} \mathrm{em}$ compósitos de QTS. 
Tabela 3. Propriedades mecânicas das membranas QTS/PVA e QTS/PVA com PMs

\begin{tabular}{lccc}
\hline Membranas & $\begin{array}{c}\text { Tensão Max. } \\
(\mathrm{MPa})\end{array}$ & $\begin{array}{c}\text { Deformação } \\
(\%)\end{array}$ & $\begin{array}{c}\text { Módulo de } \\
\text { Elastic. (MPa) }\end{array}$ \\
\hline QTS/PVA & $13,15 \pm 3,81$ & $22,8 \pm 2,6$ & $221,02 \pm 64,90$ \\
QTS/PVA-3A & $13,84 \pm 3,57$ & $12,3 \pm 1,7$ & $325,24 \pm 143,27$ \\
QTS/PVA-4A & $14,33 \pm 6,56$ & $14,7 \pm 2,5$ & $299,12 \pm 163,22$ \\
QTS/PVA-5A & $12,43 \pm 4,54$ & $15,6 \pm 2,4$ & $348,32 \pm 128,70$ \\
QTS/PVA-13X & $14,87 \pm 2,35$ & $12,0 \pm 1,7$ & $310,93 \pm 138,93$ \\
\hline
\end{tabular}

O módulo de elasticidade das membranas de QTS/PVA com PMs apresenta valores elevados, o que caracteriza o aumento na rigidez. Entretanto, considerando os elevados valores obtidos para o desvio padrão, torna-se difícil uma conclusão mais precisa. Em outro estudo reportado por Costa Jr. e Mansur, ${ }^{20}$ foi observado que o aumento do módulo de elasticidade é proporcional ao aumento do teor de QTS e do reticulante, caracterizando o aumento a fragilidade do material.

\section{Medidas de condutividade protônica e capacidade de troca iônica}

Os valores de condutividade protônica e de capacidade de troca iônica (IEC) das membranas de QTS/PVA e QTS/PVA com PMs estão listados na Tabela 4. De acordo com a literatura, o mecanismo de transporte de prótons nas membranas de QTS pode ocorrer de duas formas: o primeiro é o mecanismo de transporte pela água, onde ocorre a formação de canais de água. A combinação de prótons com moléculas de água gera conjuntos de íons hidrônios $\left(\mathrm{H}_{3} \mathrm{O}^{+}\right)$, os quais transportam os prótons através dos canais de água. ${ }^{14,19} \mathrm{O}$ segundo mecanismo, conhecido com mecanismo de Grotthuss, ocorre nas interações eletrostáticas entre os grupos amino $\left(\mathrm{NH}_{3}^{+}\right)$da QTS e os íons sulfato $\left(\mathrm{SO}_{4}^{2-}\right)$ do reticulante. Neste mecanismo, os prótons são transferidos ao longo das cadeias através das interações iônicas e ligações de hidrogênio "saltando" de um grupo funcional para outro. Neste caso, o íon $\mathrm{SO}_{4}{ }^{2-}$ reduz a barreira energética para o transporte de prótons. ${ }^{2,5,10,14,19} \mathrm{O}$ transporte dos prótons ocorre principalmente na fase QTS/PVA do que na fase PMs. A presença de partículas de PMs na membrana QTS/PVA prolonga o caminho dos prótons, conduzindo a uma redução na condutividade de prótons em relação à membrana de QTS/PVA. Entretanto, a água retida nas PMs hidrofílica promove o aumento da condutividade protônica. ${ }^{2}$ Neste estudo, os resultados apresentados na Tabela 4 mostram que as membranas de QTS/PVA com PMs tiveram os valores da condutividade protônica aumentados em relação à membrana de QTS/PVA. , $2,5,10,14^{-14}$

Os valores de IEC sugerem uma maior quantidade de grupos trocadores de íons presentes nas membranas, favorecendo o aumento da condutividade protônica, podendo ser uma aproximação indireta do potencial de condutividade protônica destas membranas. ${ }^{10} \mathrm{~A}$ membrana QTS/PVA-4A apresentou o maior valor de IEC, seguida em ordem decrescente pelas membranas QTS/PVA $>$ QTS/PVA-5A $>$ QTS/ PVA-3A $>$ QTS/PVA-13X. Este resultado corrobora com o resultado da condutividade protônica no caso da membrana com a PM 4A. Entretanto, considerando o desvio padrão, estes valores são próximos, podendo ser considerados similares. Os valores de IEC determinados corroboram com os valores reportados na literatura. ${ }^{1,2,10,14,19}$

\section{Absorção de água/metanol}

Na Tabela 4 estão apresentadas as percentagens de absorção de água e solução de metanol das membranas de QTS/PVA e QTS/PVA com PMs. A membrana de QTS/PVA absorveu percentual similar de água e de solução de metanol, sendo 3,5\% maior para a água. Isto pode estar associado a uma maior afinidade da membrana de QTS/ PVA com a água. ${ }^{1,10}$ As membranas de QTS/PVA com as PMs 3A, 4A e 5A, de maior caráter hidrofílico, apresentaram maior afinidade com a água em relação à solução de metanol, resultando numa diferença de absorção de 32, 29 e $20 \%$, respectivamente. A membrana QTS/PVA-13X apresentou afinidade similar em relação à água e à solução de metanol. Entretanto, em relação às demais PMs, ela absorveu um percentual maior para a solução de metanol (2,8\%). Este comportamento pode estar associado com o maior diâmetro médio dos poros da PM 13X, favorecendo a entrada de moléculas de metanol e, também com a maior razão Si/Al, conferindo-lhe um caráter hidrofílico menor. ${ }^{2} \mathrm{O}$ comportamento das QTS/PVA com PMs está de acordo com os resultados medidos para a condutividade protônica.

\section{CONCLUSÕES}

Foram preparadas membranas de QTS/PVA (1:1) puras e membranas de QTS/PVA (1:1) com incorporação de 12,5\% em massa das PMs 3A, 4A, 5A ou 13X e reticuladas com ácido sulfúrico para aplicação como eletrólito em PEMFC e DMFC. Estas membranas foram caracterizadas por difração de raios x, espectroscopia de infravermelho, análise térmica, microscopia eletrônica de varredura, ensaios mecânicos, condutividade protônica, capacidade de troca iônica e grau de intumescimento em água e em solução de metanol.

Os difratogramas de raio x mostraram que as distâncias interatômicas diminuem com a incorporação das PMs 4A, 5A e 13X. A maior redução ocorreu com a incorporação da PM 13X. Não foi observada variação significativa para as distâncias interatômicas entre as membranas QTS/PVA e QTS/PVA-3A, sugerindo a existência de uma mistura física. Os pequenos deslocamentos nos espectros de infravermelho das bandas em torno de 1650 e $1568 \mathrm{~cm}^{-1}$ para menores números de onda sugerem a existência de ligações de hidrogênio entre grupos hidroxila e amino da QTS e os grupos hidroxilas das PMs. Os resultados da análise térmica mostraram que a incorporação das PMs nas membranas QTS/PVA aumenta a estabilidade térmica. A maior estabilidade térmica foi verificada para a membrana de QTS/ PVA-4A. Os resultados dos ensaios mecânicos sugerem que a incorporação das PMs nas membranas QTS/PVA causa um aumento na rigidez, reduzindo a deformação. Não foram observadas variações significativas nas tensões máximas. As membranas de QTS/PVA com PMs do tipo A absorveram maiores porcentagens de água em

Tabela 4. Valores de condutividade protônica, IEC e porcentagens de absorção de água e de solução de metanol

\begin{tabular}{lcccc}
\hline Membranas & $\begin{array}{c}\text { Condutividade Protônica }(\mathrm{x} \\
\left.10^{-2} \mathrm{~S} \mathrm{~cm}^{-1}\right)\end{array}$ & $\begin{array}{c}\mathrm{IEC} \\
\left(\mathrm{mmol} \mathrm{g}^{-1}\right)\end{array}$ & $\begin{array}{c}\mathrm{H}_{2} \mathrm{O} \\
(\%)\end{array}$ & $\begin{array}{c}\mathrm{H}_{2} \mathrm{O} / \mathrm{Metanol} \\
(\%)\end{array}$ \\
\hline QTS/PVA & $(1,69 \pm 0,45)$ & $0,28 \pm 0,09$ & $73,1 \pm 2,3$ & $70,5 \pm 1,8$ \\
QTS/PVA-3A & $(2,07 \pm 0,43)$ & $0,25 \pm 0,03$ & $60,5 \pm 3,2$ & $41,2 \pm 1,7$ \\
QTS/PVA-4A & $(2,25 \pm 0,62)$ & $0,30 \pm 0,11$ & $59,3 \pm 1,7$ & $42,0 \pm 1,5$ \\
QTS/PVA-5A & $(1,98 \pm 0,55)$ & $0,23 \pm 0,09$ & $58,3 \pm 2,9$ & $47,3 \pm 2,2$ \\
QTS/PVA-13X & $(1,73 \pm 0,32)$ & $0,19 \pm 0,06$ & $55,0 \pm 2,5$ & $56,6 \pm 2,1$ \\
\hline
\end{tabular}


relação à solução de metanol. A membrana de QTS/PVA-13X absorveu porcentagens similares de água e de solução de metanol. A condutividade protônica das membranas de QTS/PVA com PMs de caráter hidrofílico (3A, 4A e 5A) e iguais razão Si/Al apresentaram valores de condutividade similares $\left(\sim 2,1 \times 10^{-2} \mathrm{~S} \mathrm{~cm}^{-1}\right)$, os quais são ligeiramente maiores em relação aos valores evidenciados para as membranas QTS/PVA e QTS/PVA-13X. A membrana de QTS/PVA$4 \mathrm{~A}$ apresentou maior valor de IEC.

\section{AGRADECIMENTOS}

Ao Conselho Nacional de Desenvolvimento Científico e Tecnológico $(\mathrm{CNPq})$ pelo apoio financeiro e ao Prof. Dr. M. M. Sá por ter cedido as peneiras moleculares.

\section{REFERÊNCIAS}

1. Wang, J.; Zhang, H.; Jiang, Z.; Yang, X.; Xiao, L.; J. Power Sources 2009, 188, 64 .

2. Wang, J.; Zheng, X.,; Wu, H.; Zheng, B.; Jiang, Z.; Hao, X.; Wang, B.; J. Power Sources 2008, 178, 9.

3. Yuan, W.; Wu, H.; Zheng, B.; Zheng, X.; Jiang, Z.; Hao, X.; Wang, B.; J. Power Sources 2007, 172, 604.

4. Smitha, B.; Devi, D. A.; Sridhar, S.; Int. J. Hydrogen Energy 2008, 33, 4138.
5. Ramíirez-Salgado, J.; Electrochim. Acta 2007, 52, 3766.

6. Smitha, B.; Sridhar, S.; Khan, A. A.; Eur. Polym. J. 2005, 41, 1859.

7. Mukoma, P.; Jooste, B. R.; Vosloo, H. C. M.; J. Power Sources 2004, $136,16$.

8. Zhong, S.; Cui, X.; Fu, T.; Na H.; J. Power Sources 2008, 180, 23,

9. Hassini-Sadrabadi, M. M.; Dashtimoghadam, E.; Majedi, F. S.; Kabiri, K.; J. Power Source 2009, 190, 318.

10. Jiang, Z.; Zheng, X.; Wu, H.; Pan, F.; J. Power Sources 2008, 185, 85

11. Yamada, M.; Honma, I.; Electrochim. Acta 2005, 50, 2837.

12. Vicentini, S. D.; Smania Jr, A.; Laranjeira, M. C. M.; Mater. Sci. Eng., C 2009, doi: 10.1016/j.msec.2009.01.026.

13. Wan, Y.; Peppley, B.; Creber, K. M. A.; Bui, V. T.; Halliop, E.; J. Power Sources 2008, 185, 183.

14. Jiang, Z.; Zheng, X.; Wu, H.; Wang, J.; Wang, Y.; J. Power Sources 2008, $180,143$.

15. Martins, L.; Cardoso, D.; Quim. Nova 2006, 29, 358.

16. Lu, L.; Sun, H.; Peng, F.; Jiang, Z.; J. Membr. Sci. 2006, 281, 245.

17. Kch, C. C.; Cavin, O. B.; McKamey, C. G.; Scarbrough, J. O.; Appl. Phys. Lett. 1983, 43, 1017.

18. Wang, Y.; Yang, D.; Zheng, X.; Jiang, Z.; Li, J.; J. Power Sources 2008, 183,454

19. Cui, Z.; Xing, W.; Liu, C.; Liao, J.; Zhang, H.; J. Power Sources 2009 , $188,24$.

20. Costa Jr., E. S.; Mansur, H. S.; Quim. Nova 2008, 31,1466. 